\title{
HOW DOES SCALE OF IMPLEMENTATION IMPACT THE ENVIRONMENTAL SUSTAINABILITY OF WASTEWATER TREATMENT INTEGRATED WITH RESOURCE RECOVERY?
}

Pablo K. Cornejo ${ }^{*}$, Qiong Zhang, James R. Mihelcic

Civil and Environmental Engineering Department, University of South Florida, 4202 E. Fowler Ave., Tampa, FL 33620, USA

*Corresponding Author: Email: pabloc@mail.usf.edu; Phone: (303)870-5171;

Fax: (813)974-2957

\section{Supporting Information}

\section{1 pages}

Table SI-1. Operating parameters and key performance metrics for systems investigated.

Table SI-2. Life cycle inventory for infrastructure of WWTPs with integrated resource recovery at different scales. Inventory items expressed per cubic meter of treated water.

Table SI-3. Life cycle inventory of operation and maintenance (O\&M) of WWTPs with integrated resource recovery at different scales. Inventory items expressed per cubic meter of treated water.

Table SI-4. Key contributors to uncertainty and sensitivity of household, community, and city systems.

Figure SI-1. Process flow diagram of household system analyzed.

Figure SI-2. Process flow diagram of community system analyzed.

Figure SI-3. Process flow diagram of city system analyzed. 


\section{Systems Investigated}

Operating parameters and key performance metrics are summarized in Table SI-1. Each scale implements different reuse and disposal methods. At the household, community, and city scale $100 \%, 77 \%$, and $56 \%$ of the treated effluent is reclaimed. At the household level all the water can be reclaimed through subsurface drip irrigation, leading to reuse for residential irrigation and de-facto aquifer recharge. At the community scale nitrogen removal is practiced since around $23 \%$ of the water is discharged to surface water during the rainy season. At the city scale deep well injection is used to inject secondary treated effluent from WWTPs deep into the confined aquifer to provide aquifer recharge and dispose of wastewater. Simplified process flow diagrams of the systems investigated are shown in Figures SI-1, SI-2 and SI-3.

Table SI-1. Operating parameters and key performance metrics for systems investigated.

\begin{tabular}{|c|c|c|c|}
\hline Parameter & Household & Community & City \\
\hline Wastewater treatment standard & $\begin{array}{l}\text { Secondary } \\
\text { biological } \\
\text { treatment for } \\
\text { subsurface drip } \\
\text { irrigation reuse }\end{array}$ & $\begin{array}{c}\text { Advanced } \\
\text { treatment with } \\
\text { nitrogen removal } \\
\text { for surface water } \\
\text { discharge \& reuse }\end{array}$ & $\begin{array}{l}\text { Advanced biological } \\
\text { treatment for reuse } \\
\text { \& deep well } \\
\text { injection }\end{array}$ \\
\hline $\mathrm{BOD}_{5}$ in treated effluent $(\mathrm{mg} / \mathrm{L})$ & $\begin{array}{c}30 \\
(20-40)^{1}\end{array}$ & $\begin{array}{c}1.8 \\
(0.8-3.5)\end{array}$ & $\begin{array}{c}2.1 \\
(1.2-2.4)\end{array}$ \\
\hline Percentage of water reclaimed $(\%)$ & 100 & 77 & 56 \\
\hline $\begin{array}{l}\text { Effluent } \mathrm{TN} \text { to soil from reclaimed } \\
\text { water }(\mathrm{mg} / \mathrm{L})\end{array}$ & $\begin{array}{c}16 \\
(2-31)\end{array}$ & $\begin{array}{c}0.23 \\
(0.03-6.8)\end{array}$ & $\begin{array}{c}2.3 \\
(1.3-3.1)\end{array}$ \\
\hline $\begin{array}{l}\text { Effluent TP to soil from reclaimed } \\
\text { water }(\mathrm{mg} / \mathrm{L})\end{array}$ & $\begin{array}{c}0.16 \\
(0.12-0.20)\end{array}$ & $\begin{array}{c}0.005 \\
(0.004-0.04)\end{array}$ & $\begin{array}{c}0.01 \\
(0.004-0.03)\end{array}$ \\
\hline Total biosolids production $(\mathrm{kg} / \mathrm{yr})$ : & $9.8^{1}$ & 60,000 & $2,894,136$ \\
\hline Water reuse pump information & $\begin{array}{c}1 \text { drip irrigation } \\
\text { pump @ } 0.70 \mathrm{HP} \\
0.26 \mathrm{hrs} / \text { day run } \\
\text { time }\end{array}$ & $\begin{array}{c}3 \text { pumps @ } \\
\text { 25,50,50 HP; } 10 \\
\text { hrs/day run time, } \\
232.6 \mathrm{TDH}\end{array}$ & $\begin{array}{c}2 \text { pumps @ } 250 \mathrm{HP} ; \\
10 \text { hrs/day run time; } \\
\text { VFD included; } \\
147.7 \mathrm{ft} \text { TDH }\end{array}$ \\
\hline
\end{tabular}

Note: Numeric values presented are average values, where values in parentheses are minimum and maximum values. 


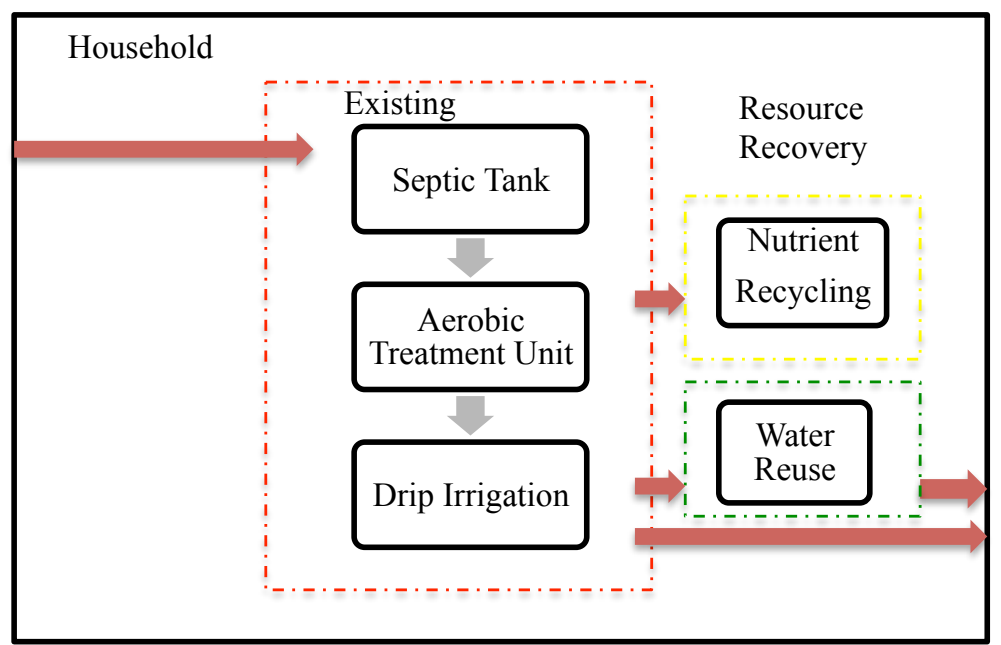

Figure SI-1. Process flow diagram of household system analyzed.

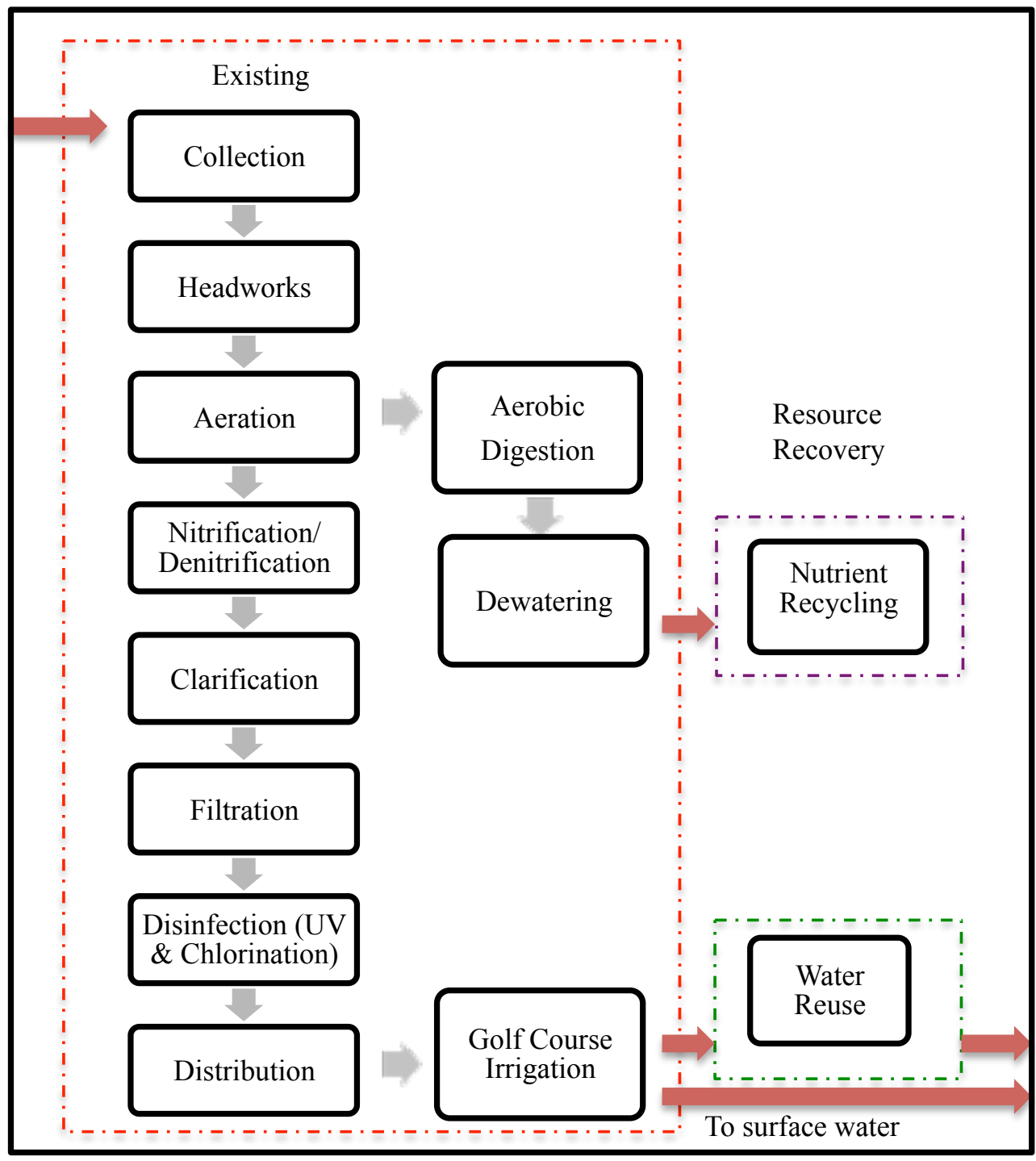

Figure SI-2. Process flow diagram of community system analyzed. 


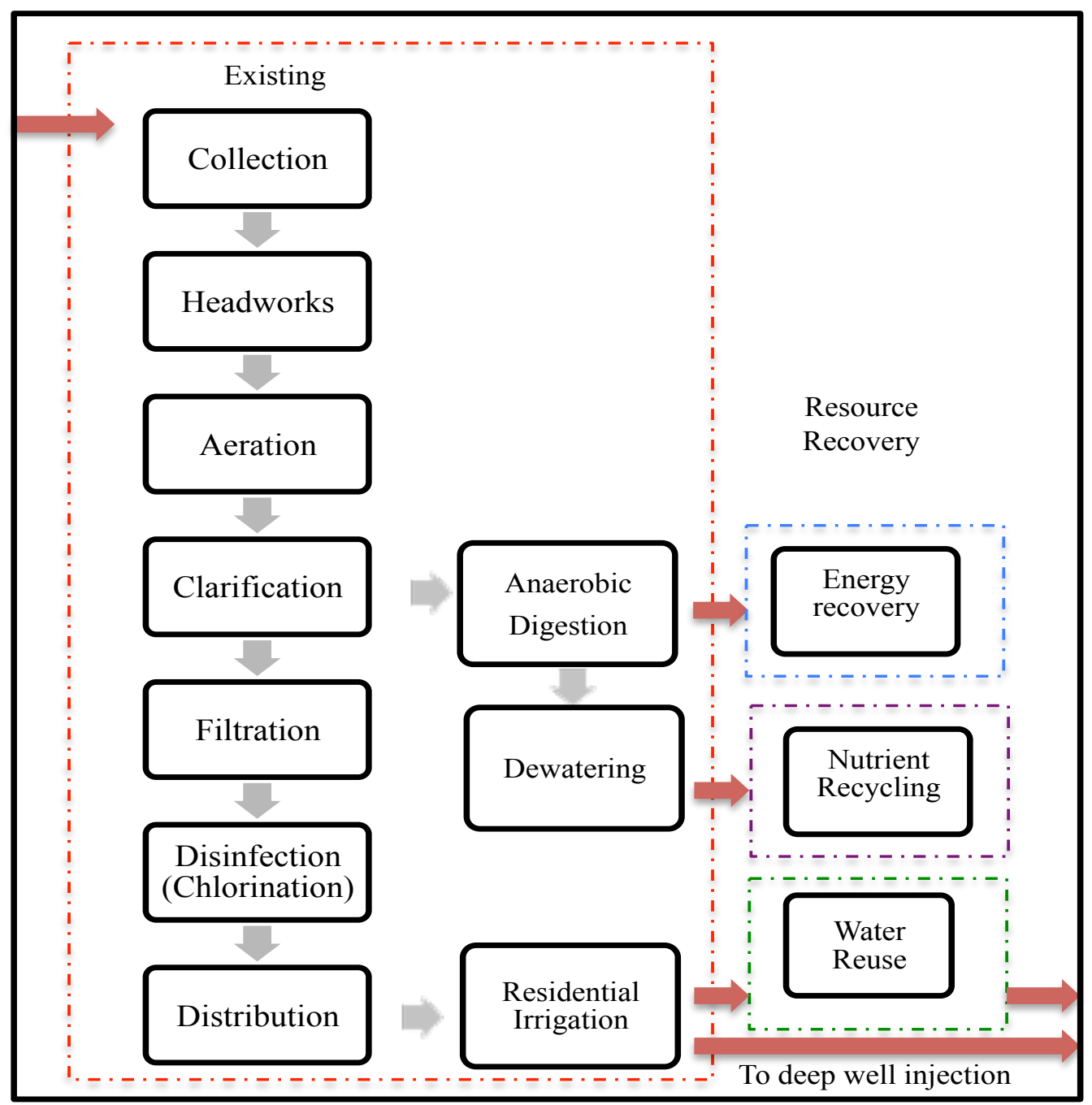

Figure SI-3. Process flow diagram of city system analyzed.

\section{Infrastructure}

Table SI-2 highlights the infrastructure life cycle inventory (LCI) for the household, community, and city systems. Data related to pipe diameter, pipe material, and pipe length was collected for wastewater collection and water reuse distribution for household, community, and city scale systems. Subsequently, pipe mass was calculated based on assumed pipe densities from various manufacturers (i.e., U.S. Plastics, Cooper Industries, Peterson Products, etc.). Collection piping for the household system was assumed to be negligible due to the short distance needed to transport wastewater to an 
on-site septic tank. Additionally, data on tank sizes and tank material were collected to estimate volumes of reinforcing steel and concrete in treatment tanks for each system. Reinforced steel was assumed to be $2 \%$ of the concrete volume, similar to water energy sustainability tool. ${ }^{2}$ Cost data on pumps, valves and fittings were collected as well, though this data was only available at the household scale and had a negligible effect on the environmental impact. Diesel consumption for excavation was assumed to have a negligible impact, because the operation and maintenance phase is the dominant contributor to the environmental impact over the life cycle. Material delivery was assumed to have a negligible impact over the life cycle, since most materials can be produced within Florida.

\section{Operation and Maintenance}

Table SI-3 highlights the operation and maintenance LCI for household, community, and city systems. Electricity data was collected from the WERF decentralized cost estimation tool ${ }^{3}$ at the household scale and directly from WWTP operators at the community and city scales. Annual average electricity usage was collected for all three systems. Annual chemical usage data was collected for the city and community scale, whereas chemicals were not used at the household scale. Biosolids removal energy and transport per cubic meter of wastewater treated were assumed to be the same at all scales, whereas operational diesel consumption per cubic meter of wastewater treated for treatment and distribution was assumed to be the same at the community and city scales.

Direct $\mathrm{CH}_{4}$ emissions from anaerobic treatment processes (e.g., septic tank at household scale, anaerobic digester at city scale), $\mathrm{N}_{2} \mathrm{O}$ emissions from nitrification 
processes, and $\mathrm{N}_{2} \mathrm{O}$ from biosolids land application were estimated using EPA and IPCC methods. ${ }^{4,5}$ Biogenic $\mathrm{CO}_{2}$ emissions were also calculated, but these emissions are considered negligible by the IPCC. ${ }^{4}$

The equation ${ }^{4}$ to calculate biogenic $\mathrm{CH}_{4}$ is:

$$
\begin{aligned}
& \mathrm{CH}_{4} \text { emission rate }\left(\mathrm{kgCH}_{4} / \mathrm{yr}\right)= \\
& 1 \mathrm{E}-3 * \mathrm{Q}_{\mathrm{ww}} * \mathrm{OD}^{*} \mathrm{Eff}_{\mathrm{OD}} * \mathrm{CF}_{\mathrm{CH}_{4}} *\left[\left(\mathrm{MCF}_{\mathrm{ww}} * \mathrm{BG}_{\mathrm{CH} 4}\right)(1-\lambda)\right]^{\mathrm{b}}
\end{aligned}
$$

Where, $10^{-3}=$ conversion from $(\mathrm{kg} / \mathrm{g}), \mathrm{Q}_{\mathrm{ww}}=$ wastewater influent flow rate $\left(\mathrm{m}^{3} /\right.$ year $)$, OD $=$ oxygen demand of influent as $\mathrm{BOD}_{5}$ or $\mathrm{COD}\left(\mathrm{g} / \mathrm{m}^{3}\right), \mathrm{Eff} \mathrm{OD}_{\mathrm{O}}=$ removal efficiency of oxygen demand, $\mathrm{CF}_{\mathrm{CH} 4}=$ conversion factor for maximum $\mathrm{CH}_{4}$ generation per unit $\mathrm{OD}(\mathrm{g} / \mathrm{gOD}), \mathrm{MCF}_{\mathrm{ww}}=$ fraction of influent $\mathrm{OD}$ converted anaerobically in wastewater treatment unit, $\mathrm{BG}_{\mathrm{CH} 4}=$ fraction of carbon as $\mathrm{CH}_{4}$ in generated biogas $(0.65)$, and $\lambda=$ biomass yield in wastewater treatment unit.

The equation to calculate $\mathrm{N}_{2} \mathrm{O}$ emissions from WWTPs is:

$$
\mathrm{N}_{2} \mathrm{O}_{\text {wwtp }}=\mathrm{Q} * \mathrm{TKN} * \mathrm{EF}_{\mathrm{N} 2 \mathrm{O}} *(44 / 28) * 1 \mathrm{E}-03
$$

Where, $\mathrm{N}_{2} \mathrm{O}_{\text {wwtp }}$ is the $\mathrm{N}_{2} \mathrm{O}$ emissions generated from WWTP process $\left(\mathrm{kg} \mathrm{N}_{2} \mathrm{O} / \mathrm{yr}\right)$ and $\mathrm{Q}$ is the wastewater influent flow rate $\left(\mathrm{m}^{3} / \mathrm{year}\right)$. Equation 2 was modified to calculate emissions per year. This equation also includes the influent TKN $(\mathrm{mg} / \mathrm{L})$, the $\mathrm{N}_{2} \mathrm{O}$ emission factor, $\mathrm{EF}_{\mathrm{N} 2 \mathrm{O}}\left(0.005 \mathrm{~g} \mathrm{~N}\right.$ emitted as $\mathrm{N}_{2} \mathrm{O}$ per $\left.\mathrm{g} T K N\right)$, and a conversion factor modified to calculate $\mathrm{kg} \mathrm{N}_{2} \mathrm{O} /$ year. $^{6}$

The $\mathrm{N}_{2} \mathrm{O}$ from land applied biosolids was calculated as follows:

$$
\mathrm{N}_{2} \mathrm{O}_{\text {biosolids }}=(44 / 28) * \mathrm{~F}_{\text {on }} * \mathrm{EF}_{1}
$$

Where, $\mathrm{N}_{2} \mathrm{O}_{\text {biosolids }}$ is the nitrous oxide generated from land applied biosolids, where $\mathrm{F}_{\mathrm{ON}}$ is the annual amount of biosolids or other additions of nitrogen applied to soils 
( $\mathrm{kg} \mathrm{N} /$ year) and $\mathrm{EF}_{1}$ is an emission factor for nitrogen additions from organic amendments as a result of the loss of soil carbon $\left(\mathrm{kg} \mathrm{N}_{2} \mathrm{O}-\mathrm{N} / \mathrm{kg} \mathrm{N}\right)$. High uncertainty is associated with $\mathrm{EF}_{1}$, where this value ranges from $0.003-0.03 .{ }^{4}$ The amount of nitrogen in biosolids was calculated by collecting the amount of biosolids hauled per year and the percent total nitrogen within the biosolids. At the city scale data on the percent of total nitrogen in biosolids was collected directly from the facility. At the household and community scale, this data was not available so a range of typical values from previous literature was used. ${ }^{1,7}$

Nutrient discharges to the environment were collected at each scale. Nitrogen and phosphorus discharges from surface water and reclaimed water to soils were collected. Additionally, nitrogen and phosphorus discharges to soil from biosolids were collected. Nutrient data were collected directly from WWTPs and typical values from previous literature were used when data was not available. ${ }^{7}$

Additionally, data on products avoided via resource recovery were collected to calculate the beneficial offsets from water reuse, nutrient recycling, and energy recovery. Potable water avoided via water reuse include chemicals and electricity offset from potable water production in Tampa, FL from a previous study. ${ }^{8}$ Fertilizers avoided via nutrient recycling assume all of the nutrients discharged in reclaimed water and biosolids replace nitrogenous and phosphorus-based fertilizers. Energy avoided via energy recovery assumes methane produced at the city scale replaces natural gas. Additional detail on this study can be found elsewhere. ${ }^{9}$ 
Table SI-2. Life cycle inventory for infrastructure of WWTPs with integrated resource recovery at different scales. Inventory items expressed per cubic meter of treated water.

\begin{tabular}{|c|c|c|c|c|}
\hline Stage & Item & Household & Community & City \\
\hline \multirow{5}{*}{$\begin{array}{l}\text { Collection } \\
\text { Infrastructure }\end{array}$} & Piping - PVC $\left(\mathrm{kg} / \mathrm{m}^{3}\right)$ & & $\begin{array}{c}0.015 \\
(0.007-0.018)\end{array}$ & 0.011 \\
\hline & Piping - VCP $\left(\mathrm{kg} / \mathrm{m}^{3}\right)$ & & & 0.188 \\
\hline & Piping - Concrete $\left(\mathrm{m}^{3} / \mathrm{m}^{3}\right)$ & & & 0.0001 \\
\hline & Piping - Reinforcing steel $\left(\mathrm{kg} / \mathrm{m}^{3}\right)$ & & & 0.013 \\
\hline & Piping - HDPE $\left(\mathrm{kg} / \mathrm{m}^{3}\right)$ & & & 0.002 \\
\hline \multirow{3}{*}{$\begin{array}{l}\text { Treatment } \\
\text { Infrastructure }\end{array}$} & Tanks - Concrete $\left(\mathrm{m}^{3} / \mathrm{m}^{3}\right)$ & $\begin{array}{c}0.0009 \\
(0.0007-0.0012)\end{array}$ & $\begin{array}{c}0.00014 \\
(0.00012-0.00016)\end{array}$ & $\begin{array}{c}0.00008 \\
(0.00007-0.00010)\end{array}$ \\
\hline & Tanks - Reinforcing steel $\left(\mathrm{kg} / \mathrm{m}^{3}\right)$ & $\begin{array}{c}0.15 \\
(0.11-0.19)\end{array}$ & $\begin{array}{c}0.022 \\
(0.018-0.026)\end{array}$ & $\begin{array}{c}0.013 \\
(0.011-0.016)\end{array}$ \\
\hline & Excavation - Diesel $\left(\mathrm{kg} / \mathrm{m}^{3}\right)$ & $\begin{array}{c}0.009 \\
(0.005-0.014) \\
\end{array}$ & & \\
\hline \multirow{12}{*}{$\begin{array}{l}\text { Water Reuse } \\
\text { Infrastructure }\end{array}$} & Piping - PVC $\left(\mathrm{kg} / \mathrm{m}^{3}\right)$ & 0.0001 & 0.002 & 0.005 \\
\hline & Piping - Cast Iron $\left(\mathrm{kg} / \mathrm{m}^{3}\right)$ & & & 0.0001 \\
\hline & Piping - Ductile Iron $\left(\mathrm{kg} / \mathrm{m}^{3}\right)$ & & & 0.07 \\
\hline & Piping - Galvanized steel $\left(\mathrm{kg} / \mathrm{m}^{3}\right)$ & & & 0.0001 \\
\hline & Piping - Steel $\left(\mathrm{kg} / \mathrm{m}^{3}\right)$ & & & 0.002 \\
\hline & Piping - Concrete $\left(\mathrm{m}^{3} / \mathrm{m}^{3}\right)$ & & & 0.00001 \\
\hline & Piping - Reinforcing Steel $\left(\mathrm{kg} / \mathrm{m}^{3}\right)$ & & & 0.002 \\
\hline & Pump Tank, Concrete $\left(\mathrm{m}^{3} / \mathrm{m}^{3}\right)$ & 0.0003 & & \\
\hline & Reinforcing steel $\left(\mathrm{kg} / \mathrm{m}^{3}\right)$ & $\begin{array}{c}0.0485 \\
(0.0476-0.0494)\end{array}$ & & \\
\hline & Pump, 12 gpm (2009USD/m³) & $\begin{array}{c}0.035 \\
(0.032-0.037)\end{array}$ & & \\
\hline & Valves $\left(2009 \mathrm{USD} / \mathrm{m}^{3}\right)$ & $\begin{array}{c}0.031 \\
(0.029-0.034)\end{array}$ & & \\
\hline & $\begin{array}{l}\text { Plastic pipe fittings }\left(2009 \mathrm{USD} / \mathrm{m}^{3}\right) \\
\text { Other fittings }\left(2009 \mathrm{USD} / \mathrm{m}^{3}\right)\end{array}$ & $\begin{array}{c}0.015 \\
(0.014-0.016) \\
0.013\end{array}$ & & \\
\hline
\end{tabular}


Table SI-3. Life cycle inventory of operation and maintenance (O\&M) of WWTPs with integrated resource recovery at different scales. Inventory items expressed per cubic meter of treated water.

\begin{tabular}{|c|c|c|c|c|}
\hline Stage & Item & Household & Community & City \\
\hline $\begin{array}{l}\text { Collection } \\
\text { O\&M }\end{array}$ & Electricity $\left(\mathrm{kWh} / \mathrm{m}^{3}\right)$ & & $\begin{array}{c}0.04 \\
(0.001-0.26)\end{array}$ & $\begin{array}{c}0.07 \\
(0.03-0.12)\end{array}$ \\
\hline \multirow{13}{*}{$\begin{array}{l}\text { Treatment } \\
\text { O\&M }\end{array}$} & Caustic Soda $\left(\mathrm{kg} / \mathrm{m}^{3}\right)$ & & & 0.002 \\
\hline & Sodium hypochlorite $\left(\mathrm{kg} / \mathrm{m}^{3}\right)$ & & & $\begin{array}{c}0.21 \\
(0.14-0.27)\end{array}$ \\
\hline & Chlorine $\left(\mathrm{kg} / \mathrm{m}^{3}\right)$ & & 0.11 & \\
\hline & Ferric sulfate $\left(\mathrm{kg} / \mathrm{m}^{3}\right)$ & & $\begin{array}{c}0.0215 \\
(0.0210-0.0219)\end{array}$ & \\
\hline & Methanol $\left(\mathrm{kg} / \mathrm{m}^{3}\right)$ & & 0.004 & \\
\hline & Polymer $\left(\mathrm{kg} / \mathrm{m}^{3}\right)$ & & $\begin{array}{c}0.009 \\
(0.006-0.012)\end{array}$ & \\
\hline & Electricity $\left(\mathrm{kWh} / \mathrm{m}^{3}\right)$ & 1.11 & 1.83 & 0.3 \\
\hline & Direct $\mathrm{CH}_{4}\left(\mathrm{~kg} \mathrm{CH} \mathrm{CH}_{4} \mathrm{eq} / \mathrm{m}^{3}\right)$ & $\begin{array}{c}0.02 \\
(0.002-0.05)\end{array}$ & - & $\begin{array}{c}0.02 \\
(0.007-0.03)\end{array}$ \\
\hline & Direct $\mathrm{N}_{2} \mathrm{O}\left(\mathrm{kg} \mathrm{CO}_{2} \mathrm{eq} / \mathrm{m}^{3}\right)$ & $\begin{array}{c}0.16 \\
(0.12-0.21)\end{array}$ & $\begin{array}{c}0.09 \\
(0.05-0.16)\end{array}$ & $\begin{array}{c}0.07 \\
(0.06-0.10)\end{array}$ \\
\hline & Direct $\mathrm{N}_{2} \mathrm{O}$ - biosolids $\left(\mathrm{kg} \mathrm{CO}_{2} \mathrm{eq} / \mathrm{m}^{3}\right)$ & $\begin{array}{c}0.003 \\
(0.001-0.01)\end{array}$ & $\begin{array}{c}0.01 \\
(0.003-0.05)\end{array}$ & $\begin{array}{c}0.05 \\
(0.01-0.23)\end{array}$ \\
\hline & Sludge removal electricity $\left(\mathrm{kWh} / \mathrm{m}^{3}\right)$ & $\begin{array}{c}4.5 \mathrm{E}-05 \\
(1.8 \mathrm{E}-05-7.2 \mathrm{E}-05)\end{array}$ & $\begin{array}{c}4.5 \mathrm{E}-05 \\
(1.8 \mathrm{E}-05-7.2 \mathrm{E}-05)\end{array}$ & $\begin{array}{c}4.5 \mathrm{E}-05 \\
(1.8 \mathrm{E}-05-7.2 \mathrm{E}-05)\end{array}$ \\
\hline & Sludge removal transport $\left(\mathrm{tkm} / \mathrm{m}^{3}\right)$ & $\begin{array}{c}0.0023 \\
(0.0021-0.0027)\end{array}$ & $\begin{array}{c}0.0023 \\
(0.0021-0.0027)\end{array}$ & $\begin{array}{c}0.0023 \\
(0.0021-0.0027)\end{array}$ \\
\hline & Diesel $\left(\mathrm{kg} / \mathrm{m}^{3}\right)$ & & $\begin{array}{c}0.016 \\
(0.10-0.43)\end{array}$ & $\begin{array}{c}0.016 \\
(0.10-0.43)\end{array}$ \\
\hline \multirow{2}{*}{$\begin{array}{l}\text { Water Reuse } \\
\text { O\&M }\end{array}$} & Electricity $\left(\mathrm{kWh} / \mathrm{m}^{3}\right)$ & 1.4 & 0.5 & 0.20 \\
\hline & Diesel $\left(\mathrm{kg} / \mathrm{m}^{3}\right)$ & & $\begin{array}{c}0.025 \\
(0.0004-0.28)\end{array}$ & $\begin{array}{c}0.025 \\
(0.0004-0.28)\end{array}$ \\
\hline \multirow{6}{*}{$\begin{array}{c}\text { Direct } \\
\text { Nutrients to } \\
\text { Environment }\end{array}$} & $\mathrm{N}$ to surface water $\left(\mathrm{g} / \mathrm{m}^{3}\right)$ & & $\begin{array}{c}0.65 \\
(0.34-4.93)\end{array}$ & \\
\hline & $P$ to surface water $\left(\mathrm{g} / \mathrm{m}^{3}\right)$ & & $\begin{array}{c}0.13 \\
(0.02-0.77)\end{array}$ & \\
\hline & $\mathrm{N}$ to soil from water reuse $\left(\mathrm{g} / \mathrm{m}^{3}\right)$ & $\begin{array}{c}16.4 \\
(2.0-30.8)\end{array}$ & $\begin{array}{c}0.2 \\
(0.03-6.8)\end{array}$ & $\begin{array}{c}2.3 \\
(1.3-3.1)\end{array}$ \\
\hline & P to soil from water reuse $\left(\mathrm{g} / \mathrm{m}^{3}\right)$ & $\begin{array}{c}0.16 \\
(0.12-0.20)\end{array}$ & $\begin{array}{c}0.005 \\
(0.004-0.04)\end{array}$ & $\begin{array}{c}0.01 \\
(0.004-0.03)\end{array}$ \\
\hline & $\mathrm{N}$ to soil for biosolids $\left(\mathrm{g} / \mathrm{m}^{3}\right)$ & $\begin{array}{c}0.3 \\
(0.04-0.8)\end{array}$ & $\begin{array}{c}1.3 \\
(0.2-3.0)\end{array}$ & $\begin{array}{c}4.5 \\
(0.7-12.6)\end{array}$ \\
\hline & P to soil from biosolids $\left(\mathrm{g} / \mathrm{m}^{3}\right)$ & $\begin{array}{c}0.014 \\
(0.008-0.027)\end{array}$ & $\begin{array}{c}0.06 \\
(0.04-0.10)\end{array}$ & $\begin{array}{c}0.09 \\
(0.06-0.15)\end{array}$ \\
\hline \multirow{6}{*}{$\begin{array}{l}\text { Products } \\
\text { Avoided via } \\
\text { Resource } \\
\text { Recovery }\end{array}$} & Potable water avoided $\left(\mathrm{MJ} / \mathrm{m}^{3}\right)$ & 7.17 & 5.55 & 4.03 \\
\hline & $\mathrm{N}$ fertilizer avoided- water reuse $\left(\mathrm{g} / \mathrm{m}^{3}\right)$ & $\begin{array}{c}30.0 \\
(20.0-40.0)\end{array}$ & $\begin{array}{c}1.1 \\
(0.2-6.9)\end{array}$ & $\begin{array}{c}8.7 \\
(4.0-13.2)\end{array}$ \\
\hline & $\mathrm{P}$ fertilizer avoided- water reuse $\left(\mathrm{g} / \mathrm{m}^{3}\right)$ & $\begin{array}{c}8.0 \\
(6.0-10.0)\end{array}$ & $\begin{array}{c}0.2 \\
(0.1-1.4)\end{array}$ & $\begin{array}{c}0.6 \\
(0.2-1.4)\end{array}$ \\
\hline & $\mathrm{N}$ fertilizer avoided- biosolids $\left(\mathrm{g} / \mathrm{m}^{3}\right)$ & $\begin{array}{c}0.65 \\
(0.42-1.03)\end{array}$ & $\begin{array}{c}3.0 \\
(2.1-3.9)\end{array}$ & $\begin{array}{c}10.4 \\
(7.2-16.3)\end{array}$ \\
\hline & P fertilizer avoided- biosolids $\left(\mathrm{g} / \mathrm{m}^{3}\right)$ & $\begin{array}{c}0.71 \\
(0.39-1.37)\end{array}$ & $\begin{array}{c}3.2 \\
(1.9-5.1)\end{array}$ & $\begin{array}{c}4.6 \\
(2.8-7.4)\end{array}$ \\
\hline & Energy avoided-natural gas $\left(\mathrm{kg} / \mathrm{m}^{3}\right)$ & & & $0.02(0.01-0.03)$ \\
\hline
\end{tabular}




\section{Uncertainty and Sensitivity Analysis}

Key contributors to the uncertainty of the environmental impact categories investigated in this study were assessed using Monte-Carlo uncertainty analysis. Additionally, the model's sensitivity to key input parameters were evaluated. Table SI-4 shows the standard deviation of key contributors to uncertainty and sensitivity for the household, community, and city systems investigated.

Table SI-4. Key contributors to uncertainty and sensitivity for household, community, and city systems.

\begin{tabular}{clcc}
\hline Impact Category & Item & $\begin{array}{c}\text { Standard } \\
\text { Deviation }\end{array}$ & $\begin{array}{c}\text { Sensitivity } \\
\text { Factor }\end{array}$ \\
\hline \multirow{3}{*}{ Eutrophication } & $\mathrm{N}$ to soil (water reuse) - Household & 3.5 & $0.4^{\mathrm{a}}$ \\
Potential & $\mathrm{P}$ to surface water - Community & 0.7 & 0.06 \\
& $\mathrm{~N}$ to soil (biosolids) - Community & 0.3 & 0.09 \\
& $\mathrm{~N}$ to soil (biosolids) - City & 1.4 & $0.2^{\mathrm{a}}$ \\
\hline \multirow{5}{*}{ Embodied Energy } & Treatment Tanks - Household & 0.4 & 0.04 \\
& Collection Electricity - Community & 0.9 & 0.008 \\
& Water Reuse Diesel - City & 4.5 & 0.05 \\
\hline \multirow{5}{*}{ Carbon footprint } & Direct CH - Household & 0.3 & 0.07 \\
& Collection Electricity - Community & 0.1 & 0.008 \\
& Treatment Chemicals - City & 0.04 & $0.1^{\mathrm{a}}$ \\
\hline
\end{tabular}

${ }^{a}$ High sensitivity values

Eutrophication potential results were sensitive to $\mathrm{N}$ to soil (water reuse) at the household system and $\mathrm{N}$ to soil (biosolids) at the city system, whereas sensitivity was lower for key input parameters at the community system. For embodied energy, results were found to have a low sensitivity to key input parameters at all scales. Lastly, carbon footprint results were found to have a low sensitivity to key input parameters for the household and community systems, but a higher sensitivity to treatment chemicals for the city system. 


\section{References}

(1) Asano, T.; Burton, F. L.; Leverenz, H. L.; Tsuchihashi, R.; Tchobanoglous, G. Water Reuse Issues, Technologies, and Applications; McGraw-Hill: New York, 2007.

(2) Water Energy Sustainability Tool. http://west.berkeley.edu (accessed March 2016)

(3) Decentralized systems performance and costs fact sheets. Website; http://www.werf.org/i/c/DecentralizedCost/Decentralized_Cost.aspx

(4) Waste. In 2006 IPCC Guidelines for National Greenhouse Gas Inventories, Vol. 5; Eggleston, H.S., Buendia, L., Miwa, K., Ngara, T., \& Tanabe, K., Eds.; IGES: Japan, 2006.

(5) Greenhouse Gas Emissions Estimation Methodologies for Biogenic Emissions from Selected Source Categories: Solid Waste Disposal, Wastewater Treatment, Ethanol Fermentation; U.S. Environmental Protection Agency: Research Triangle Park, N.C., 2010.

(6) Greenhouse Nitrogen Emissions from Wastewater Treatment Operations: Interim Report; Report No. U4R07a; Water Environment Research Foundation (WERF): Alexandria, VA, 2010.

(7) Tchobanoglous, G.; Burton, F.L.; Stensel, H.D. Wastewater Engineering, Treatment and Reuse, fourth edition, international edition; McGraw-Hill: New York, 2004.

(8) Santana, M. V. E.; Zhang, Q.; Mihelcic, J. R. Influence of water quality on the embodied energy of drinking water treatment. Environ. Sci. Technol., 2014, 48 (5), 3084-3091.

(9) Cornejo, P.K. Environmental Sustainability of Wastewater Treatment Plants Integrated with Resource Recovery: The Impact of Context and Scale. Ph.D. Dissertation, University of South Florida, Tampa, FL, 2015. http://scholarcommons.usf.edu/etd/5669/ (accessed March 2016) 